\title{
Validation of the New International League Against Epilepsy 2017 Classification for Determining Seizure Type in Indian Patients
}

\author{
Sandhya Manorenj ${ }^{1} \quad$ Navya Sagari ${ }^{2}$ \\ ${ }^{1}$ Department of Neurology, Princess Esra Hospital, Deccan College \\ of Medical Sciences, Hyderabad, Telangana, India \\ 2IMO Department of Neurology, ESIC Medical College, Hyderabad, \\ Telangana, India \\ Address for correspondence Sandhya Manorenj, MBBS, DNB, \\ Department of Neurology, Princess Esra Hospital, Deccan \\ College of Medical Sciences, Hyderabad, Telangana, India \\ (e-mail: drsandhyamanorenj@gmail.com).
}

Int J of Epilep 2021;7:29-35.

\begin{abstract}
Introduction Epilepsy is a common condition in neurology comprising several electroclinical syndromes and seizure disorders of varying known and unknown etiologies that require variable diagnostic workup, treatment, and have obviously different prognoses. Therefore, for appropriate patient management, the best possible classification system for epilepsy is required. The International League Against Epilepsy (ILAE) is continuously working on this with the latest classification provided in 2017. There is little knowledge about seizure type based on newer classification systems in Indian patients.

Aims and Objective To test the applicability of the newer ILAE 2017 classification of epilepsy in determining seizure type in Indian patients, with respect to right patient management, the best classification system for epilepsy is necessary.

Materials and Methods Prospective data of 310 consecutive patients with seizures presenting in neurology department was collected from December 2017 to June 2018 and analyzed according to the newer systems of classification of seizures proposed by ILAE in 2017.

Results All 310 patients in age ranging from one year to 72 years with seizures could be classified according to the ILAE 2017 classification system. Focal onset seizure was noted in 66 patients (21.3\%), while 244 patients $(78.7 \%$ ) had generalized onset based on clinical onset of seizure. Awareness was impaired in 262 (84.5\%) patients. Motor onset seizure was observed in 278 patients (89.6\%), while nonmotor seizure included absence, sensory, cognitive, and autonomic seizures.

Keywords

- seizures

- epilepsy

- classifications

Conclusion The present study showed that all patients could be classified using ILAE 2017 classification system. Majority of seizure were generalized onset, predominantly motor type of seizure with impaired awareness using clinical description of classifying seizure, while focal onset seizure was the majority type of seizure when ancillary information was considered.
\end{abstract}

published online July 20, 2021
DOI https://doi.org/

$10.1055 / \mathrm{s}-0041-1731934$ ISSN 2213-6320 (c) 2021. Indian Epilepsy Society.

This is an open access article published by Thieme under the terms of the Creative Commons Attribution-NonDerivative-NonCommercial-License, permitting copying and reproduction so long as the original work is given appropriate credit. Contents may not be used for commercial purposes, or adapted, remixed, transformed or built upon. (https://creativecommons.org/licenses/by-nc-nd/4.0/).

Thieme Medical and Scientific Publishers Pvt. Ltd. A-12, 2nd Floor, Sector 2, Noida-201301 UP, India 


\section{Introductions}

Epilepsy is one of the prevailing neurological diseases leading to significant medical and social morbidity. The disturbances of neuronal activity that occur during seizures may result in motor (convulsions, abnormal posturing, and loss of tone) and nonmotor (strange sensations, behavior change, emotions, loss of memory) symptoms that can either be or not be associated with loss of consciousness. ${ }^{1}$ It is believed there are more than 12 million persons with epilepsy (PWE) in India. ${ }^{2}$ Its prevalence is estimated to be $1 \%$ of our population and is greater in the rural (1.9\%) compared with the urban population (0.6\%). Epilepsy can occur at any age and affects both sexes.

The characterization of seizures, epilepsies, and epilepsy conditions makes a system, for clinicians, scientists, patients, and their families. This characterization has developed throughout the years since 1981 onward, and in 2017 , the International League Against Epilepsy (ILAE) distributed an operational arrangement of seizures and epilepsies. Understanding this characterization is significant in the analysis, treatment, and comprehension of seizures and epilepsies, including epilepsy frequency. The 2017 ILAE classification builds on new developed definitions of seizures and epilepsy. ${ }^{3}$ Accordingly, seizure classification begins by determining whether the initial manifestations of the seizure are focal or generalized. If the onset of the seizure is incomprehensible or unclear, the seizure is of unknown onset. Focal seizures are classified in keeping with the individual's level of awareness, the foremost outstanding motor or nonmotor options of the seizure, and whether the ictus evolves to a bilateral tonic-clonic seizure. ${ }^{4}$ Similarly, generalized seizures are classified in keeping with motor or nonmotor manifestations. Motor seizures can be either tonic-clonic or alternative motor seizures. Absence seizure was classified under generalized nonmotor seizure, according to this system. There is a three-tier system in ILAE 2017 classification: first, to identify seizure type, then to diagnose the epilepsy type; finally, figuring whether it is an epilepsy syndrome where a specific syndrome diagnosis can be made. The new classification incorporates etiology along each stage. An etiology of epilepsy is broadly divided into six categories, namely, structural, infective, genetic, immune, metabolic, and unknown origin. $^{5}$

This new system appears to be more systematic, as entities are straightforward and precise. We tried to classify seizure type in our patients who presented to our department. Through this study, we have attempted to test the validation of this new system in a developing country like India with restricted resources.

\section{Materials and Methods}

\section{Study Design}

Consecutive patients with new onset seizure or with earlier history of seizure or epilepsy, who were attending regular follow-up to neurology epilepsy clinic outpatient and inpatient departments of ESIC Medical College and
Superspeciality Center, Hyderabad, were identified for this study. The study was conducted from December 2017 to June 2018 and patients having relevant investigations, including at least one good brain imaging (CT or MRI) and one EEG ictal or interictal, were enrolled in the study.

\section{Participants}

Patients who had presented during the above-mentioned study period with either seizure in past or present, willing to give detailed history, and willing to get investigated were included in the study. Information was gathered utilizing a standard proforma to record the factors, including demographic information, history of febrile seizures, positive family ancestry of epilepsy, etiological elements, objective neurological findings, seizure types, EEG and neuroradiology reports, metabolic workup, and genetic studies whenever done. Participants were analyzed, according to the three levels proposed by ILAE 2017 by a qualified neurologist. Classification of seizure in participants was initially done, based on the basic version of the classification given by ILAE 2017; later, participants were grouped to seizure type, based on the expanded version of the seizure type. ${ }^{5}$ For accurate classification of seizure, onset of seizure was compared with ancillary information like EEG and MRI brain. With further information or future observed seizures, a reclassification of unknown-onset seizures into focal or generalized-onset categories was possible. According to the etiological factors, participants were grouped into six main categories. These included structural, infective, metabolic, immunogenic, genetic, and unknown causes. Telephonic survey for recurrence of seizure was done at 2 years.

\section{New Definitions Used in Study According to ILAE $2017^{4}$}

1. Epilepsy: A disease of the brain defined by any of the following conditions: (1) at least two unprovoked (or reflex) seizures occurring > 24 hours apart; (2) one unprovoked (or reflex) seizure and a probability of further seizures similar to the general recurrence risk (at least 60\%) after two unprovoked seizures, occurring over the next 10 years; (3) diagnosis of an epilepsy syndrome.

2. Awareness: knowledge of self or environment.

3. Emotional seizures: seizures presenting with an emotion or the appearance of having an emotion as an early prominent feature, such as fear, spontaneous joy or euphoria, laughing (gelastic), or crying (dacrystic).

4. Focal onset bilateral tonic-clonic seizure: a seizure type with focal onset and an awareness or impaired awareness, either motor or nonmotor, progressing to bilateral tonic-clonic activity. The prior term was seizure with partial onset along with secondary generalization.

5. Unclassified seizure: referring to a seizure type that cannot be described by the ILAE 2017 classification either because of inadequate information or unusual clinical features. If the seizure is unclassified, because the type of onset is unknown, a limited classification may still be derived from observed features. 


\section{Statistical Analysis}

Data obtained in the study was subjected to statistical analysis with SPSS (Version18.0, IBM). Variables were represented in the form of counts and percentages. Comparison of variables between adults and pediatric age group was calculated using percentage of proportion differences between them, and $p$-value was estimated. $p$-value of less than 0.05 was considered statistically significant.

\section{Ethic Statement}

Information obtained in this study was collected prospectively, with the approval of the local ethics committee. Participants gave their informed consent prior to enrollment in the study.

\section{Results}

\section{Baseline Characteristics}

A total of 310 patients were enrolled in the study, comprising 176 male and 134 female. Age range of the participants were from 1 year to 72 years. Mean age of the patients were 24.4 \pm 15.36 years. As many as $118(38.06 \%)$ patients belonged to the pediatric age group ( $\leq 15$ years).

\section{Seizure Type (Level 1 of ILAE 2017)}

Initially, while trying to classify seizures, 22 patients' (7.09\%) seizures were unwitnessed at first onset of seizure. With further information and later observed seizures, a reclassification of unknown onset seizure into focal or generalized onset categories was possible. We classified patients into seizure types, based on clinical description obtained from eyewitness and video (33\%) brought in by family members as supportive evidence, even though that evidence is not part of the classification. We found that in $33 \%$ of cases, evidence of seizure was recorded in video. Out of these $33 \%$, focal seizure was noted in $17 \%$ cases. Based on eye witness and video evidence, we could classify the seizure type in all the 310 (100\%) patients using 2017 ILAE classification system. Patients could be classified into either focal onset or generalized onset seizure type. As many as 66 (21.3\%) patients had clinically focal onset seizure, while 244 (78.7\%) patients had generalized onset seizure. Focal onset seizure with preserved awareness was observed in 44 (14.19\%) patients, while 22 (7.09\%) patients had focal onset seizure with impaired awareness. Among the focal onset seizure, 44 (66.6\%) patients had motor onset, 18 (27.2\%) patients had nonmotor onset, and 4 (6.06\%) patients had focal to bilateral tonic-clonic seizure. Among generalized onset seizure, 228 (93.4\%) patients had motor onset, while 16 (6.5\%) patients had nonmotor (absence) onset seizure. Among motor generalized seizure, tonic-clonic type was observed in $192(84.2 \%)$ patients and other motor types noted in 36 (15.7\%) patients (-Fig. 1).

Based on ILAE 2017 expanded version of classification of seizure, the majority of focal seizure were focal unaware tonic seizure, seen in 18 (27.27\%) patients, followed by focal aware tonic seizure seen in 16 (24.24\%) patients ( - Fig. 2 ). Of the 244 total patients of generalized seizure, majority had generalized tonic seizure (GTS), noticed among 114 (46.72\%)
Frequency of seizure type (Basic version classification)

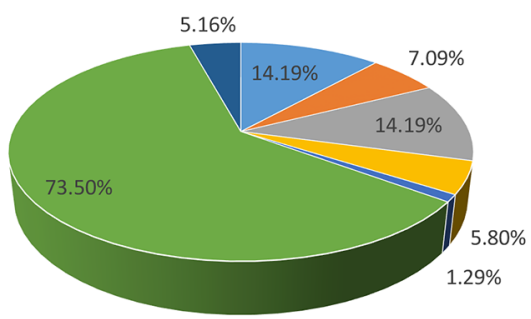

- Focal onset with preserved awarenss

Focal onset with impaired awarenss

- Focal motor seizure

- Focal non motor seizure

- Focal to bilateral tonic -clonic seizure

- Generalized onset motor seizure

- Generalized onset non-motor seizure

Fig. 1 Showing frequency of seizure type using basic version classification of ILAE 2017. ILAE, International League Against Epilepsy.

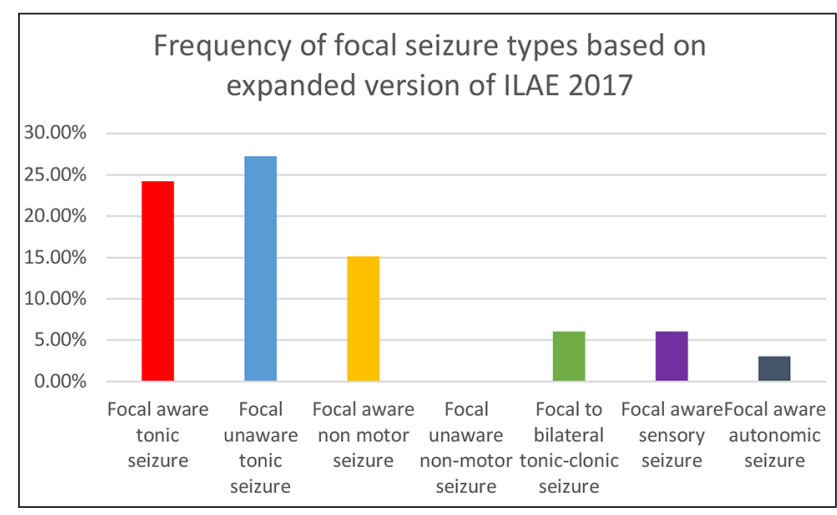

Fig. 2 Showing frequency of focal seizure type, based on expanded version of ILAE 2017 classification. ILAE, International League Against Epilepsy.

patients, followed by generalized tonic-clonic seizure (GTCS), observed in 108 (44.26\%) patients ( - Fig. 3 ).

Seizure classification based on onset of seizure (historical and eyewitness), either focal or generalized as mentioned above, was later compared with ancillary information like EEG and MRI brain for accurate classification of seizure and diagnosis of seizure type. We found that some cases, who were classified under generalized onset of seizure, had either EEG or MRI brain showing focality. Such cases were later reclassified broadly as focal seizure and focal as bilateral tonic-clonic seizure. Reclassification of seizure type, including onset and ancillary information, showed change in type of seizure, with focal onset seizure observed in 176 patients (56.7\%) and generalized seizure in 134 patients (43.2\%). Focal to bilateral tonic-clonic seizure was observed in 94 cases (30.3\%) (-Fig. 4).

\section{Epilepsy Type (Level 2 of ILAE 2017)}

As per ILAE 2017, after analysis of the seizure type (Level 1), the subsequent stage is determination of epilepsy type (level 2), based on the definition of epilepsy previously referenced. Out of total 310 patients, epilepsy was diagnosed in 240 patients (77.41\%), while 70 (22.5\%) patients 


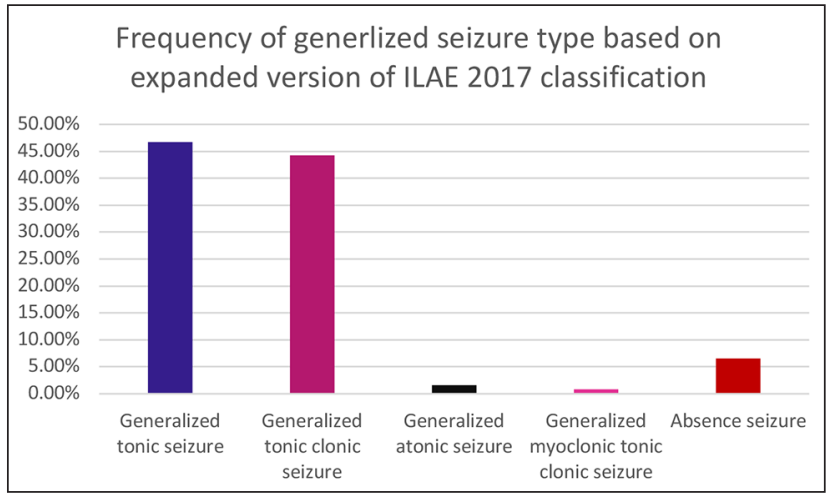

Fig. 3 Generalized seizure type classification and its frequency, based on expanded version of ILAE 2017. ILAE, International League Against Epilepsy.

had presented with single episode of unprovoked seizure. Hence, epilepsy was unclassified in $22.5 \%$ cases. The majority of those diagnosed with epilepsy had generalized epilepsy (60\%) (- Table 1). We found mismatch in epilepsy type, based on clinical onset alone versus combination of clinical description and ancillary evidence (EEG and MRI brain).

\section{Epilepsy Syndrome (Level 3 of ILAE 2017)}

Among the 310 patients, 90 patients were diagnosed to have an epilepsy syndrome having an aggregate of features which incorporated seizure types, EEG, and imaging features (-Fig. 5). EEG abnormality was observed in 186 (60\%) patients. Among them, 56 (62.22\%) patients had generalized epilepsy syndrome. We could confirm focality in EEG in four cases belonging to the focal seizure group, who were already classified as focal seizure, based on clinical history and evidence.

\section{Etiology}

Among the six etiological groups already described earlier, the majority of patients had infective (27.9\%) etiology of seizure, followed by structural lesions ( - Table 2). On comparing etiology of seizure between pediatric and adults ( $\mathbf{- T a b l e ~} \mathbf{3}$ ), the majority of children had genetic cause of seizure, and this difference was significant $(p<0.0001)$ However, altogether 86 patients' cause of seizure was unknown, and difference between pediatric and adult age group was significant $(p$-value $=0.0009)$.

We reviewed our records regarding focality of seizure using imaging finding and found that 84 patients $(27 \%)$ had unilateral focal structural lesion and 92 patients (29.6\%) had multifocal lesions in brain. Hence, altogether, we expect 176 patients to have focal seizure. This accounts for $56.7 \%$ of cases to have focal onset of seizure. Based on clinical description, $21.2 \%$ cases had focal seizure, while MRI evidence showed additional 35.5\% to have focal onset seizure, even though there is no eyewitness account of any focality of seizures

We tried to follow cases after 2 years of initial study using telephonic conversation; 15 (4.8\%) patients did not have phone number, so could not be contacted, and in the remaining 38\% cases, there was no response. As much

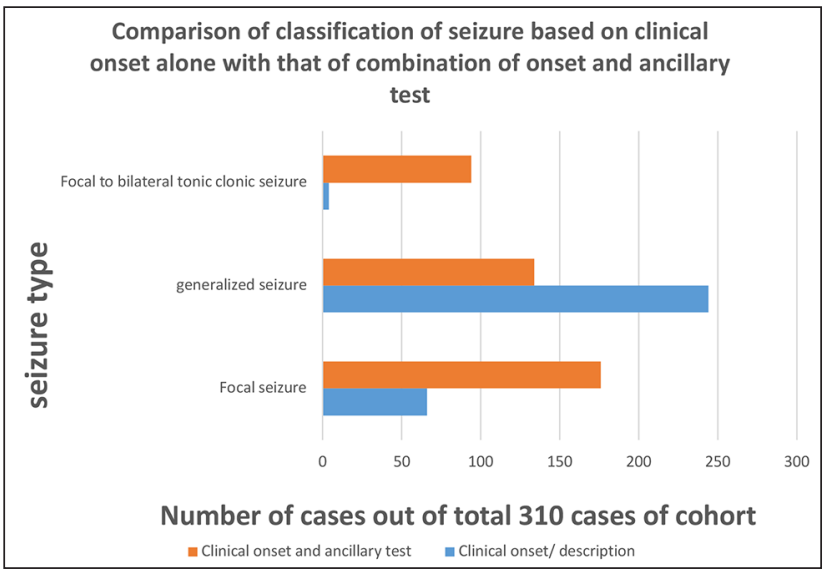

Fig. 4 Comparison of classification of seizure based on clinical onset alone with that of combination of clinical onset and ancillary tests (eg: EEG and MRI of brain)

Table 1 Showing type of epilepsy (level 2 of ILAE 2017 classification)

\begin{tabular}{|c|c|c|}
\hline $\begin{array}{l}\text { A: Epilepsy } \\
\text { type based on } \\
\text { clinical onset/ } \\
\text { description alone }\end{array}$ & Number $(n=310)$ & Frequency (\%) \\
\hline Focal epilepsy & 48 & 15.48 \\
\hline $\begin{array}{l}\text { Generalized } \\
\text { epilepsy }\end{array}$ & 186 & 60 \\
\hline $\begin{array}{l}\text { Combined focal } \\
\text { and generalized } \\
\text { epilepsy }\end{array}$ & 6 & 1.9 \\
\hline $\begin{array}{l}\text { Unclassified } \\
\text { epilepsy } \\
\text { (single unprovoked } \\
\text { Seizure) }\end{array}$ & 70 & 22.5 \\
\hline $\begin{array}{l}\text { B: Epilepsy type } \\
\text { based on combined } \\
\text { clinical onset/ } \\
\text { description and } \\
\text { EEG and MRI brain } \\
\text { abnormality }\end{array}$ & Number $(n=310)$ & Frequency (\%) \\
\hline $\begin{array}{l}\text { Focal epilepsy } \\
\text { (EEG or MRI show- } \\
\text { ing focality) }\end{array}$ & 176 & 56 \\
\hline $\begin{array}{l}\text { Generalized } \\
\text { epilepsy } \\
\text { (EEG abnormal } \\
\text { showing general- } \\
\text { ized seizure and } \\
\text { MRI brain normal }\end{array}$ & $\begin{array}{l}50 \text { (8 excluded as } \\
\text { EEG was normal) }\end{array}$ & 16.1 \\
\hline $\begin{array}{l}\text { Combined focal } \\
\text { and generalized } \\
\text { epilepsy }\end{array}$ & 6 & 1.9 \\
\hline $\begin{array}{l}\text { Unclassified } \\
\text { epilepsy } \\
\text { (included gen- } \\
\text { eralized seizure } \\
\text { with normal EEG } \\
\text { and normal MRI, } \\
\text { single seizure, EEG } \\
\text { inconclusive) }\end{array}$ & 78 & 25.2 \\
\hline
\end{tabular}

Abbreviation: ILAE, International League Against Epilepsy. 


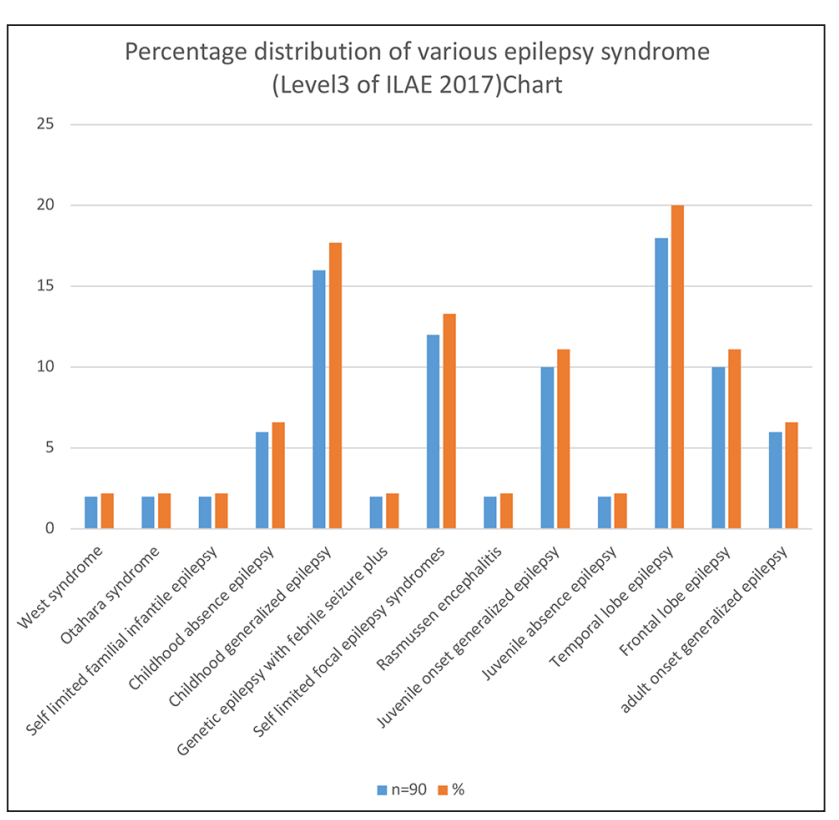

Fig. 5 Chart showing percentage distribution of various epilepsy syndromes.

as $57.16 \%$ responded to questions posed to either patient or close caregivers. Recurrence of seizure was observed in $68 \%$ due to withdrawal of drugs.

\section{Discussion}

The new classification of epilepsy is a multilevel classification, designed to categorize epilepsy into distinct clinical environments. We tried to validate this newer system in our patients afflicted with seizure. Our study group chiefly consisted of adults and few pediatric age group patients. This is because the study was performed at the department of adult neurology. All the patients could be classified into the various seizure types, either focal onset or generalized onset seizure, based on level 12017 ILAE classification system. Epilepsy could not be diagnosed among the entire study group using level 2 system of ILAE 2017 classification. This difference is because patients with single seizures were also included in the study, and the study period was short. We could classify the majority of our patients (77.5\%) into one of the epilepsy types, namely, focal epilepsy, generalized epilepsy, and combined focal and generalized epilepsy. As much as $22.5 \%$ of the patients were diagnosed to have unclassified epilepsy, based on the presence of single unprovoked seizure, as the diagnosis of epilepsy was made, based on the presence of two or more unprovoked seizures. Of the patients with generalized onset seizure, who were classified as generalized epilepsy, based on definition for epilepsy, eight $(0.02 \%)$ patients had a normal EEG. This group of patients was diagnosed clinically as generalized epilepsy, but EEG was normal and MRI brain was normal, and they were grouped under unclassified epilepsy for accurate classification. We could not find similar study validating the applicability of this revised newer classification system and its applicability at all the three levels. Literature review showed that in one study, the authors tried to explore the long-term applicability of the new ILAE definition of epilepsy by following patients with single unprovoked seizure for a period of 10 years and concluded $15.1 \%$ patients were newly diagnosed with epilepsy after a single seizure. ${ }^{6}$ In our study, we could not label single unprovoked seizure as epilepsy, since follow-up was short.

We tried to identify an epilepsy syndrome using level 3 ILAE 2017 system of classification. Relevant selection of an epilepsy syndrome was done, based on seizure type, EEG finding, neuroimaging features, and family history, for a particular age of onset of epilepsy. Our patients with epilepsy could be broadly grouped into focal epilepsy or generalized epilepsy syndrome; however, we had some difficulties in classifying individual syndrome, according to the age. Out of total patients, 220 patients could not be classified as specific epilepsy syndrome. This is probably because the majority of the patients had single EEG and $60 \%$ of the EEG were normal. Another reason could be that in adult population, epilepsy syndromes are contrarily rare. There are scarce clinical studies from India regarding specific epilepsy syndrome. Literature review showed single clinical study from India, where authors attempted to determine the applicability of ILAE 2010 classifications for epilepsies. In their study, only $19.6 \%$ patients could be classified as specific electroclinical syndrome, while in the present study using ILAE 2017 system, we could classify $29.3 \%$ patients as specific epileptic syndromes. ${ }^{7}$ On comparing these percentage proportion differences between both studies, we found a significant difference $(p$-value $=0.0015,95 \%$ CI $3.6451 \%$ to $15.9089 \%$ ) and a higher documentation of epilepsy syndrome using the latest ILAE system. This could be due to higher awareness and advanced technology for diagnosing epilepsy syndrome.

The moment the patient presents with first epileptic seizure, as clinicians, our aim was to determine the etiology of the patient's epilepsy. This was done at each level of ILAE 2017 classification system. We could not establish etiology in $86(27.74 \%)$ of our patients. This could be due to lack of availability of genetic testing, which is scarce in developing country like India, or unaffordability of these tests. Another reason was that we could not subject our patients to SPECT or PET scan if higher resolution imaging brain was normal. Hence, we might have missed many immune-mediated epilepsies. We found infection as the major cause of epilepsy followed by structural etiology. These findings were similar to previous studies reported from India., ${ }^{8}$ We tried to subdivide patients into pediatric and adult group and found

Table 2 Showing etiology of epilepsy

\begin{tabular}{|l|l|l|}
\hline $\begin{array}{l}\text { Etiological } \\
\text { groups }\end{array}$ & $\begin{array}{l}\text { Numbers }(\boldsymbol{n}= \\
\mathbf{3 1 0})\end{array}$ & Percentage (\%) \\
\hline Structural & 78 & 25.16 \\
\hline Infection & 84 & 27.09 \\
\hline Metabolic & 10 & 3.22 \\
\hline Immune & 0 & 0 \\
\hline Genetic & 52 & 16.77 \\
\hline Unknown & 86 & 27.74 \\
\hline
\end{tabular}


Table 3 Comparison between etiology of seizure and type of seizure among pediatric and adult age groups using ILAE 2017 classification system

\begin{tabular}{|l|l|l|l|}
\hline Etiology of seizure & $\begin{array}{l}\text { Children age } \leq 15 \text { years } \\
n=118(\%)\end{array}$ & Adults $\boldsymbol{n}=192(\%)$ & $p$-Value \\
\hline Structural causes & $32(27.11)$ & $46(23.95)$ & 0.5 \\
\hline Infection & $26(22.03)$ & $58(30.2)$ & 0.11 \\
\hline Metabolic & $4(3.3)$ & $6(3.12)$ & 0.93 \\
\hline Immune & 0 & 0 & 0 \\
\hline Genetic & $36(30.5)$ & $16(8.33)$ & $<0.0001$ \\
\hline Unknown & $20(16.94)$ & $66(34.37)$ & 0.0009 \\
\hline Type of seizure & $14(11.86)$ & $46(23.95)$ & \\
\hline \multicolumn{1}{|l|}{ Focal seizure } & $102(86.44)$ & $142(73.95)$ & 0.0090 \\
\hline Generalized seizure & $2(1.69)$ & $4(2.08)$ & 0.0092 \\
\hline $\begin{array}{l}\text { Focal to bilateral tonic } \\
\text {-clonic type }\end{array}$ & & 0.80 \\
\hline
\end{tabular}

Abbreviation: ILAE, International League against Epilepsy.

that genetic etiology was predominant in children, while infective etiology dominated among adults with seizure. In a similar study from north India where majority of patients were adults, infective etiology was the predominant cause of seizure. ${ }^{7}$ Higher proportion of children had genetic etiology when compared with adults, and this difference was significant ( $p$-value $<0.0001$ ). Among the adults, we could not determine the etiology in 66 patients, while in children, etiology was unknown only in 20 cases, and this difference was significant $(p$-value $=0.0009$ ). This could be because, in view of economic constraints, workup for autoimmune epilepsy was lacking in the majority of our adult patients. Although $>50 \%$ of our patients had structural and infective etiologies, in that we expected focal onset bilateral tonic-clonic seizure, but generalized seizure was the major type of seizure. These is due to the fact that multiple brain lesions were noted in the form of multiple calcified granuloma, bilateral subcortical white matter lesion, and meningoencephalitis, so the history obtained would have described them as generalized seizure. Second, we could not do serial EEG to localize the multifocal seizure type in such cases. We found a mismatch with clinical classification alone versus with EEG/MRI data. It was initially found that generalized seizure type accounted for majority of seizure type (78.7\%), based on clinical description alone, while combining MRI evidence and EEG showed that $56.7 \%$ should have focality of seizure, even though there is no eyewitness of focality. It is likely to be there in the practical scenario. Hence, combining clinical description and ancillary information, majority of seizure type was focal seizure type, of which majority (53.4\%) had focal to bilateral tonic-clonic seizure. Second, in generalized seizure with EEG showing abnormality (generalized epileptiform discharges) perfectly belong to generalized seizure or epilepsy, but those with generalized seizure and EEG and MRI brain normal can be grouped into unclassified epilepsy for accurate classification. In the present study, seizure type could be classified in all cases as either focal, generalized, or unknown onset. Among unknown onset subsequent seizure, ancillary studies helped to classify them as either focal or generalized seizure type. However, epilepsy was unclassified in $22.5 \%$ cases, as they had single seizure in their lifetime and did not have two or more unprovoked seizures to label as epilepsy; follow-up was for a short period. We could not label these cases as epilepsy, although we know the type of seizure based on clinical history, video and EEG; moreover, few cases of generalized epilepsy were not conclusive. Hence, combination of clinical description, onset, subsequent seizure type, and EEG and MRI of brain would help in accurate classification of seizure and higher level of diagnostic certainty. Long-term follow-up of cases helps in classifying unclassified epilepsy to classified epilepsy.

\section{Limitations}

Limitations of the study included being conducted in a single center, and it was a cross-sectional study. Sample size was small, and we could not establish epilepsy in cases with single unprovoked seizure due to short follow-up period. Fewer cases were labeled as epilepsy syndrome in view of normal EEG at baseline. Genetic, antineural antibodies and autoimmune workup could not be done in all cases due to financial constraints; hence, etiology remained unknown in one-third cases. Even though type of seizure classification as mentioned in ILAE 2017 is based on clinical description, MRI of brain and EEG has shown additional cases to have focality of seizure despite the absence of eyewitnesses.

To our knowledge, ours is the first study that has tested the applicability of the revised ILAE 2017 classification systems among Indians. The recent ILAE seizure and epilepsy classification system focused on designing a framework in order to better classify seizures and epilepsies. This has been practically proved in our study; we could classify seizure type in $100 \%$ of our cases and epilepsy type in $77.5 \%$ of our patients. The newer system also highlights the etiology of seizure, which would lead to a better understanding of 
incidence of epilepsy and its treatment. However, we could not identify etiology of epilepsy in all our patients.

\section{Conclusion}

Using the newer system of classification, we could classify all our patients into various seizure types. Generalized seizure type with motor involvement and impaired awareness was the most common type of seizure noted in our patients. Focal onset seizure remained masked using only clinical description. We could not classify epilepsy in one-third of our cases using this system due to single unprovoked seizure at presentation.

\section{Funding}

None.

\section{Conflict of Interest}

None declared.

\section{Acknowledgment}

The authors acknowledge the contribution of Miss Sharon, a biotechnology resident.

\section{References}

1 Giourou E, Stavropoulou-Deli A, Giannakopoulou A, Kostopoulos GK, Koutroumanidis M, Introduction to Epilepsy and Related Brain Disorders. Cyberphysical Systems for Epilepsy and Related Brain Disorders. Switzerland: Springer International Publishing; 2015

2 Amudhan S, Gururaj G, Satishchandra P. Epilepsy in India I: epidemiology and public health. Ann Indian Acad Neurol 2015;18(3):263-277

3 Fisher RS, Cross JH, French JA, et al. Operational classification of seizure types by the international league against epilepsy: position paper of the ILAE commission for classification and terminology. Epilepsia 2017;58(4):522-530

4 Fisher RS, Cross JH, D'Souza C, et al. Instruction manual for the ILAE 2017 operational classification of seizure types. Epilepsia 2017;58(4):531-542

5 Scheffer IE, Berkovic S, Capovilla G, et al. ILAE classification of the epilepsies: position paper of the ILAE Commission for Classification and Terminology. Epilepsia 2017;58(4):512-521

6 Beretta S, Carone D, Zanchi C, et al. PRO-LONG Study Group. Long-term applicability of the new ILAE definition of epilepsy. Results from the PRO-LONG study. Epilepsia 2017;58(9):1518-1523

7 Uttam AK, Joshi R, Dwivedi R, et al. Applicability of the new ILAE classification for epilepsies (2010) in persons with epilepsy at a tertiary care center in India. Epilepsia 2013;54(4):751-756

8 Sinha S, Satishchandra P, Mahadevan A, Bhimani BC, Kovur JM, Shankar SK. Fatal status epilepticus: a clinico-pathological analysis among 100 patients: from a developing country perspective. Epilepsy Res 2010;91(2-3):193-204

9 SinghG,BawaJ,Chinna D, etal. Association between epilepsyand cysticercosis and toxocariasis: a population-based case-control study in a slum in India. Epilepsia 2012;53(12):2203-2208 